\title{
Therapieentscheidung
}

\section{Evidenz und eigene Erfahrung zählen auch bei der Phytotherapie}

\author{
Wonach richten sich Ärzte bei der Therapieentscheidung? Im Wesentli- \\ chen nach den Kriterien der evidenzbasierten Medizin (EbM) sagt eine \\ kürzlich durchgeführte, online-basierte Befragung. Dabei umfasst EbM \\ mehr als nur die Erkenntnisse systematischer Forschung.
}

$\mathrm{V}$ iele Faktoren beeinflussen das Verordnungsverhalten von Ärzten. Neben Wirksamkeit und Verträglichkeit spielen auch die Erfahrung des Arztes, die Erwartungshaltung der Patienten oder Kosten eine Rolle. Eine Forderung, die in jüngerer Zeit an Gewicht gewonnen hat, ist die Evidenz-Basierung der getroffenen Maßnahme, quasi die auf „Beweismaterial“ gestützte Entscheidung.

Welchen Stellenwert diese Forderung für Ärzte in der Praxis tatsächlich hat, was sie darunter verstehen und ob sie diese Maßstäbe auch an die Verordnung von Phytotherapeutika anlegen, war Gegenstand einer Erhebung des Unternehmens coliquio.

\section{Wirksamkeit steht an erster Stelle}

Um herauszufinden, welches die primären Kriterien für eine Therapieentscheidung sind, wurden exemplarisch 120 Allgemeinmedizinern und $\mathrm{HNO}$-Ärzten zunächst allgemeine Fragen zu Arzneimitteln und dem persönlichen Verordnungsverhalten gestellt. Aus den Antworten wurde deutlich: Das mit Abstand wich- tigste Kriterium für die Verordnung oder Empfehlung eines Arzneimittels ist dessen Wirksamkeit (74\%), gefolgt von seiner Verträglichkeit (51 \%).

\section{Erfahrung und Studien - beides zählt}

Doch wie lässt sich aus Sicht der Ärzte Wirksamkeit nachweisen? Welche Faktoren halten sie für entscheidend? Eine wichtige Rolle spielen hier klinische (placebokontrollierte, doppelblinde) Studien. Für knapp ein Viertel der Befragten sind sie das ausschlaggebende Merkmal. 15\% verlassen sich lieber auf ihre eigenen positiven Erfahrungen. Für die Mehrheit ist jedoch die Kombination aus beidem der entscheidende Maßstab. Entsprechend hielten $43 \%$ der Teilnehmer eine Arznei dann für wirksam, wenn es Belege aus Wissenschaft und eigener Erfahrung gibt.

David Sackett, einer der Urväter der evidenzbasierten Medizin, würde sich über dieses Ergebnis freuen, entspricht die Einschätzung der Ärzte doch weitgehend seinem Konzept von EbM: Die Integration individueller klinischer Expertise (Arzterfahrung) mit der bestmögli- chen externen Evidenz aus systematischer Forschung.

So weit, so gut. Doch gelten dieselben Motive auch bei der Empfehlung von Phytotherapeutika oder stehen hier alleine Verträglichkeit, Patientenwunsch und Praxisbudget im Fokus der Überlegungen? Wohl kaum. Vielmehr erhielt ein in derselben Umfrage vorgestelltes Konzept zur Evidenzbasierung der Phytotherapie hohe Zustimmungswerte. $94 \%$ der Teilnehmer sind der Meinung, dass Phytotherapie EbM sein kann, 53 \% unterstützen diese Aussage sogar „auf jeden Fall“.

Das Fazit dieser Befragung von Allgemeinmedizinern und HNO-Ärzten: Für die Verordnung von Arzneimitteln ist die Wirksamkeit nach EbM-Kriterien für Ärzte entscheidend. Darunter verstehen sie in der Mehrheit die Integration der gegenwärtig besten externen, wissenschaftlichen Evidenz mit der Erfahrung des behandelnden Arztes. Die Phytotherapie macht hier keine Ausnahme; auch sie kann nach Einschätzung der Ärzte Evidenz basierte Medizin sein - vorausgesetzt Wirksamkeit, Erfahrung, Qualität und Datenlage stimmen. Dr. Christoph Posch

Survey Konzept-Test, online-gestützte Befragung von 60 Allgemeinmedizinern und 60 HNO-Ärzten, coliquio $\mathrm{GmbH}$, Konstanz im September 2012

\section{Hier steht eine Anzeige.}

